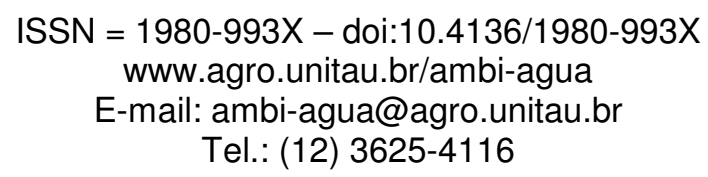

\title{
Macroinvertebrados aquáticos do reservatório do rio Batalha para a captação das águas e abastecimento do município de Bauru, SP, Brasil (doi:10.4136/ambi-agua.87)
}

\author{
Fabio Laurindo da Silva ${ }^{1}$; Jandira Liria Biscalquini Talamoni ${ }^{2}$; Gabriel Lucas Bochini ${ }^{2}$; \\ Sonia Silveira Ruiz ${ }^{3}$; Diana Calcidoni Moreira ${ }^{2}$ \\ ${ }^{1}$ Universidade Federal de São Carlos, UFSCar \\ E-mail: fabelha@hotmail.com \\ ${ }^{2}$ Universidade Estadual Paulista, UNESP \\ E-mail: \{talamoni, bochini\}@fc.unesp.br; diana@ hotmail.com \\ ${ }^{3}$ Universidade Paulista, UNIP \\ E-mail: ssruiz@fc.unesp.br
}

\section{RESUMO}

Neste estudo a composição e diversidade de macroinvertebrados aquáticos foram avaliadas no reservatório de captação das águas do rio Batalha para tratamento e abastecimento do município de Bauru, SP. As coletas foram realizadas nos períodos de seca (junho a agosto/2005) e de chuva (dezembro/2005 a fevereiro/2006). Foram identificados 840 organismos distribuídos em oito táxons (oito durante a estação seca e quatro na estação chuvosa). O sistema apresentou baixa abundância e diversidade de organismos provavelmente em resposta à qualidade da água do reservatório e às variações químicas e físicas desta, associadas à ocorrência de chuvas.

Palavras-chave: Variáveis físicas e químicas; qualidade da água; bioindicadores.

\section{Aquatic macroinvertebrates of Batalha river reservoir for water captation and supply of the city of Bauru, SP, Brazil}

\begin{abstract}
In this study the composition and diversity of aquatic macroinvertebrates were evaluated in the reservoir of water captation of Batalha river for treatment and supplying of the city of Bauru. The samples were collected in dry (from June to August, 2005) and rainy (from December, 2005 to February, 2006) seasons. We analyzed and identified 840 organisms belonging to 8 taxa in dry season and 4 taxa in rainy season. The system presented low abundance and diversity of macroinvertebrates probably due to the water quality and its physical and chemical variations associated with rain events.
\end{abstract}

Keywords: Physical and chemical conditions; water quality; bioindicators.

\section{INTRODUÇÃO}

A construção de reservatórios artificiais de água, que visam à produção de energia e ao abastecimento urbano, produz várias alterações nos sistemas hidrológico, atmosférico, biológico e social nas regiões em que são construídos e nas áreas atingidas. Ao interceptar o fluxo de um rio, além das alterações causadas ao longo da bacia hidrográfica, nota-se também 
a interferência da represa na evolução das comunidades de organismos aquáticos como peixes, macrófitas, plâncton e de bentos (Tundisi, 1992 e Dumont, 1999).

Os macroinvertebrados aquáticos têm sido amplamente utilizados como bioindicadores de qualidade de água (Roque e Trivinho-Strixino, 2000; Fonseca-Gessner e Guereschi, 2000; Kleine e Trivinho-Strixino, 2005). De acordo com Callisto et al. (2005), os bioindicadores, em ecossistemas hídricos são organismos cuja presença, quantidade e distribuição indicam a dimensão dos impactos ambientais e permitem a avaliação integrada dos efeitos ecológicos causados por múltiplas fontes de poluição. Nesse contexto, os macroinvertebrados destacamse por estarem relacionados com o enriquecimento orgânico dos corpos aquáticos e pela eficácia na detecção de perturbações antrópicas em função de algumas de suas características: baixa motilidade, grande abundância, alta longevidade dos organismos e baixo custo dos métodos empregados em seu estudo, além da fácil implementação e obtenção de informações, que podem ser sumarizadas e interpretadas por não especialistas (Queiroz et al., 2000; Roque e Trivinho-Strixino, 2000).

A comunidade de macroinvertebrados está representada por vários filos: Arthropoda, insetos, ácaros, crustáceos; Mollusca, gastrópodos e bivalves; Annelida, oligoquetos e sanguessugas; Nematoda e Platyhelminthes (Hauer e Resh, 1996). Entre estes, os insetos destacam-se pela diversidade e abundância (Lake, 1990), sendo sua distribuição relacionada às características morfométricas, físicas e químicas do habitat, à disponibilidade de recursos alimentares e ao hábito das espécies (Merritt e Cummins, 1996).

O Rio Batalha, pertencente à Bacia Hidrográfica do Médio Tietê Superior, nasce na Serra da Jacutinga (Município de Agudos, SP) e abrange, total ou parcialmente, os municípios de Agudos, Bauru, Piratininga, Avaí, Duartina, Gália, Presidente Alves, Reginópolis e Uru, percorrendo um trajeto de $167 \mathrm{~km}$ de extensão e desaguando no rio Tietê. A má utilização e ocupação do solo, pelas atividades antrópicas desenvolvidas na sua área de drenagem (monocultura, reflorestamento e pecuária), têm reduzido a mata nativa e ripícola, desencadeando alterações da qualidade de suas águas e exposição da área das nascentes a crescentes processos de erosão de áreas terrestres adjacentes ao rio que conduzem ao assoreamento de seu leito, além da poluição de suas águas (Talamoni, 1999). Na tentativa de manter a capacidade da represa constante e minimizar o assoreamento, o reservatório tem sido submetido a dragagens periódicas do fundo, o que tem causado alterações nas características daquele sistema.

O objetivo deste estudo foi analisar a composição da comunidade de macroinvertebrados aquáticos do reservatório de captação das águas do rio Batalha no município de Bauru (SP) e a influência das variáveis físicas e químicas da água sobre a abundância desses organismos nas estações seca e chuvosa.

\section{MATERIAL E MÉTODOS}

O reservatório no qual é feita a captação das águas do rio Batalha está situado no limite

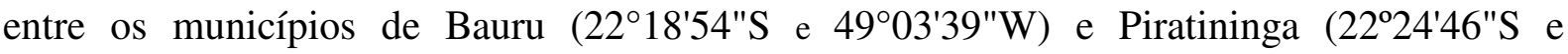
$49^{\circ} 08^{\prime} 05^{\prime \prime W}$ ), na região centro-oeste do estado de São Paulo (Figura 1). O reservatório foi construído em 1943, e sua ampliação data de 1967, tendo havido uma nova expansão da área alagada em agosto de 2004. Com uma capacidade média diária de captação de $500 \mathrm{~L} / \mathrm{seg} / \mathrm{dia}$ e vazão de exploração de $1.800 \mathrm{~m}^{3} / \mathrm{h}$, o reservatório atualmente possui formato irregular com comprimento que pode variar de 200 a $270 \mathrm{~m}$, largura de 15 a $200 \mathrm{~m}$ e profundidade de 0,60 $\mathrm{m}$ a 2,00 m, segundo informações obtidas na ETA-DAE/Bauru. O sistema em questão possui formato irregular, com vegetação ciliar apenas em um pequeno trecho das margens. 
SILVA, F. L.; TALAMONI, J. L. B.; BOCHINI, G. L.; RUIZ, S. S.; MOREIRA, D. C. Macroinvertebrados aquáticos do reservatório do rio Batalha para a captação das águas e abastecimento do município de Bauru, SP, Brasil. Ambi-Agua, Taubaté, v. 4, n. 2, p. 66-74, 2009. (doi:10.4136/ambi-agua.87)

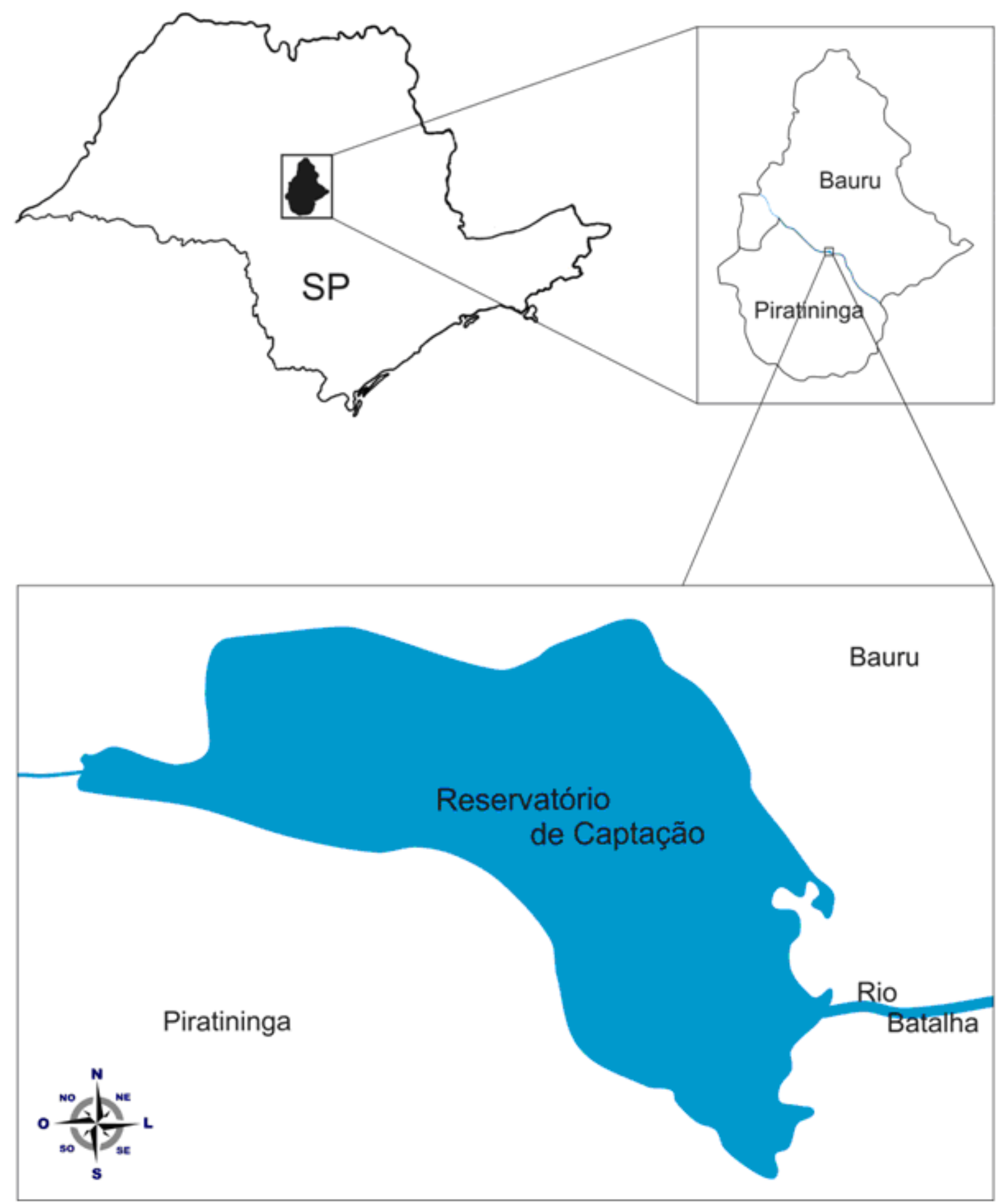

Figura 1. Mapa dos municípios de Bauru e Piratininga (SP), evidenciando o reservatório do rio Batalha para a captação das águas e abastecimento.

Para este estudo foram realizadas coletas de sedimento em dois períodos, visando à obtenção de dados que caracterizassem a qualidade da água e os macroinvertebrados aquáticos presentes no ambiente durante as estações seca (junho a agosto/2005) e chuvosa (dezembro/2005 a fevereiro/2006). O sedimento foi coletado em seis unidades amostrais mensais, com auxílio de um pegador do tipo Ekman-Birge $\left(225 \mathrm{~cm}^{2}\right)$, sendo obtido um total de 18 unidades amostrais por período de estudo. As amostragens ocorreram em dois pontos da represa, um localizado na região central do reservatório, na zona profundal, e outro, na zona litoral. Em cada ponto foram coletadas, seriadamente na mesma unidade espacial, três sub- 
amostras, que corresponderam à área do pegador. Em laboratório, o sedimento foi lavado em uma peneira com malha de rede de $200 \mu \mathrm{m}$. A fauna retida na peneira passou por uma triagem e foi preservada em etanol $70 \%$ para ser posteriormente identificada com auxílio de bibliografia específica (Brinkhurst e Marchese, 1989; Merrit e Cummins, 1996; Fernández e Dominguez, 2001).

A fim de se conhecer a composição e diversidade da comunidade de macroinvertebrados aquáticos, após a identificação, os espécimes foram enumerados e analisados segundo a participação relativa de cada táxon. Foi determinada a riqueza de espécies $(\mathrm{S})$, a diversidade de Shannon (H') e a uniformidade de Pielou (J), de acordo com metodologia em Pinto-Coelho (2002).

A avaliação da qualidade das águas foi feita mediante a análise de algumas variáveis abióticas. Para tal, antes da amostragem de sedimento foram coletadas duas amostras de água mensais, a uma profundidade de $1 \mathrm{~m}$, em cada um dos pontos de coleta, totalizando 12 amostras por período de estudo, essas medições foram realizadas em horário similar. Os resultados são apresentados sob a forma de valores médios. A temperatura $\left({ }^{\circ} \mathrm{C}\right)$ e o pH da água foram determinados in situ, com auxílio de um termômetro de mercúrio de $0,5^{\circ} \mathrm{C}$ de precisão e um medidor Corning - $\mathrm{pH} 30$, respectivamente. A condutividade elétrica da água $(\mu \mathrm{S} / \mathrm{cm})$ também foi determinada in situ, utilizando-se um medidor Corning - CD-55. A concentração de oxigênio dissolvido $(\mathrm{mg} / \mathrm{L})$ foi determinada em laboratório, por meio do método de Winkler (Golterman et al., 1978). Os valores relativos à dureza da água $\left(\mathrm{mgCaCO}_{3} / \mathrm{L}\right)$ foram obtidos por titulometria, com utilização do método descrito no Standard Methods for the Examination of Waters and Wastewaters (Apha, 1995). As concentrações de amônia e nitrito, assim como as de nitrato, foram determinadas em laboratório, segundo as metodologias descritas por Mackereth et al. (1978) e por Rodier (1975), respectivamente.

\section{Resultados e Discussão}

Neste estudo foram registrados 8 táxons de macroinvertebrados aquáticos no reservatório de captação das águas do rio Batalha, sendo que 4 táxons foram comuns aos dois períodos de amostragem (Tabelas 1 e 2). No período seco, o táxon que apresentou maior abundância de indivíduos foi Chironomidae, com 157 indivíduos, seguido por Tubificidae (152), Naididae (119), Ceratopogonidae (68), Gastropoda (16) Polymitarcyidae (9), Glossiphoniidae (2) e Nematoda (1). No período chuvoso, a família Tubificidae foi a que apresentou maior número de espécimes (292). Nesse período também foi constatada a ocorrência de Naididae, Chironomidae e Gastropoda, com apenas 10, 8 e 6 indivíduos, respectivamente.

Os resultados comparativos da análise faunística demonstraram diferenças entre as abundâncias de organismos observadas nos diferentes períodos (Tabela 2). Verifica-se que foram obtidos maiores valores totais de indivíduos durante o período seco (524 indivíduos), quando comparados com o período chuvoso (316 indivíduos). Essa diferença possivelmente pode ser explicada pela maior estabilidade encontrada no substrato bentônico durante o período de estiagem, uma vez que este pode ser afetado pela ocorrência de fortes chuvas no período chuvoso, já que o reservatório em questão apresenta dimensão reduzida, o que possivelmente o torna ainda mais sujeito às alterações físicas impostas pela influência pluvial.

De acordo com Fusari (2006), as larvas de Chironomidae e os Oligochaeta são grupos que se destacam na fauna bentônica de sedimentos lênticos, por participarem no aspecto estrutural e funcional da comunidade seja por sua abundância, conforme pôde ser confirmado neste estudo, ou pela biomassa. 
SILVA, F. L.; TALAMONI, J. L. B.; BOCHINI, G. L.; RUIZ, S. S.; MOREIRA, D. C. Macroinvertebrados aquáticos do reservatório do rio Batalha para a captação das águas e abastecimento do município de Bauru, SP, Brasil. Ambi-Agua, Taubaté, v. 4, n. 2, p. 66-74, 2009. (doi:10.4136/ambi-agua.87)

Tabela 1. Abundância absoluta $(\mathrm{N})$ e relativa (ni) da comunidade de macroinvertebrados aquáticos do rio Batalha no reservatório de captação, nos períodos seco e chuvoso.

\begin{tabular}{|c|c|c|c|c|}
\hline \multirow{2}{*}{ Táxon } & \multicolumn{2}{|c|}{ Período Seco } & \multicolumn{2}{|c|}{ Período Chuvoso } \\
\hline & $\mathrm{N}$ & $\mathrm{Ni}$ & $\mathrm{N}$ & $\mathrm{ni}$ \\
\hline Diptera & & & & \\
\hline Ceratopogonidae & 69 & 0,129 & & \\
\hline Chironomidae & 157 & 0,299 & 8 & 0,025 \\
\hline Ephemeroptera & & & & \\
\hline Polymitarcyidae & 9 & 0,017 & & \\
\hline $\begin{array}{c}\text { Gastropoda } \\
\text { Hirudinea }\end{array}$ & 16 & 0,031 & 6 & 0,019 \\
\hline Glossiphoniidae & 2 & 0,004 & & \\
\hline $\begin{array}{l}\text { Nematoda } \\
\text { Oligochaeta }\end{array}$ & 1 & 0,002 & & \\
\hline Naididae & 119 & 0,227 & 10 & 0,032 \\
\hline Tubificidae & 152 & 0,290 & 292 & 0,924 \\
\hline
\end{tabular}

Com base na comparação dos resultados obtidos nos períodos seco e chuvoso, é possível observar que os números de indivíduos de Tubificidae e Chironomidae, por exemplo, não só foram maiores durante o período seco, mas que para os Chironomidae esse aumento foi de cerca de 20 vezes, o que significa, provavelmente, um incremento de matéria orgânica no sistema, dado que esses organismos são considerados tolerantes à poluição, e, portanto, servem como indicadores de má qualidade da água (Johnson et al., 1993). Também o desaparecimento de Ceratopogonidae, Glossiphoniidae, Nematoda e Polymitarcyidae no período chuvoso pode indicar que a estação chuvosa aumenta, sazonalmente, o impacto das atividades antrópicas sobre o reservatório.

Essas variações de abundância entre os táxons, nos diferentes períodos de amostragem, estão de acordo com os resultados obtidos por Rodrigues (1997) em seu estudo realizado na Represa do Beija-Flor (Município de Luís Antônio, SP), quando o autor também observou uma maior abundância de organismos durante os meses de seca, tendo atribuído o fato à maior estabilidade (nível de oxigênio dissolvido, homogeneidade de substrato e transparência) encontrada no substrato bentônico no período seco, uma vez que fortes chuvas podem afetá-lo, pois devido às reduzidas dimensões do reservatório, a comunidade de macroinvertebrados aquáticos torna-se mais sujeita às modificações físicas impostas pela influência das chuvas.

Tabela 2. Número de táxons (S), número de indivíduos (N), índice de diversidade de Shannon (H’) e uniformidade $(\mathrm{J})$ da comunidade de macroinvertebrados aquáticos do rio Batalha no reservatório de captação, nos períodos seco e chuvoso.

\begin{tabular}{l|cccc}
\hline & S & N & H'$^{\prime}$ & J \\
\hline Período Seco & 8 & 524 & 1,53 & 0,74 \\
Período Chuvoso & 4 & 316 & 0,35 & 0,25 \\
\hline Total & 8 & 840 & & \\
\hline
\end{tabular}

Segundo Shimizu (1978), a maior densidade de Tubificidae (Oligochaeta) durante o período chuvoso pode estar relacionada com os valores mais elevados de temperatura atmosférica, essa variável pode provocar a depleção do oxigênio dissolvido na água e favorecer os tubifícideos, tolerantes a baixas concentrações de oxigênio. A fauna de 
Tubificidae pôde ser favorecida também pela maior aporte de material alóctone para o sistema aquático durante o período chuvoso. Resultados semelhantes foram constados por Fusari (2006) na Represa do Fazzari e por Brandimarte (1991) na Represa de Paraibuna, ambas localizadas no Estado de São Paulo.

É importante mencionar que neste estudo houve o estabelecimento de condições mais favoráveis à fauna bentônica oportunista (Chironomidae e Oligochaeta) no sistema aquático, provavelmente associado à ocorrência de assoreamento e consequente aumento de matéria orgânica, mudanças na natureza do substrato e alterações hidrológicas (Talamoni, 1999). O aumento da concentração de matéria orgânica (Strixino e Trivinho-Strixino, 1998), as mudanças na natureza do substrato (Cohen, 1986) e as alterações hidrológicas (Dumnicka, 1987) são citadas entre as principais características que permitem o aumento da densidade desses organismos, como ocorreu neste estudo.

De acordo com Lindergaard (1995), em geral, as represas comportam uma diversidade de fauna bentônica reduzida quando comparada à de outros sistemas hídricos. Neste estudo, os valores obtidos para os índices de diversidade e de uniformidade foram baixos nos dois períodos de amostragem (Tabela 2). Isso se se deve ao fato de as famílias Chironomidae (Diptera) e Tubificidae (Oligochaeta) terem apresentado elevada abundância, enquanto as demais apresentaram abundâncias reduzidas (Glossiphoniidae e Nematoda, no período seco, e Gastropoda, no período chuvoso).

Com relação às variáveis físicas e químicas analisadas no reservatório de captação das águas do rio Batalha (Tabela 3), no período de estiagem foram registrados valores mais elevados de fosfato $(1,9 \mathrm{mg} / \mathrm{L})$, oxigênio dissolvido $(6,0 \mathrm{mg} / \mathrm{L})$, condutividade elétrica $(227,5$ $\mu \mathrm{S} / \mathrm{cm})$ e alcalinidade $(102,2 \mathrm{mg} / \mathrm{L})$ da água em comparação com os do período chuvoso, quando essas variáveis, incluindo a dureza da água $(80,2 \mathrm{mgCaCO} / \mathrm{L})$, foram menores e, de certa forma, podem ter influenciado a composição da fauna de macroinvertebrados aquáticos. Para o pH e a amônia, no entanto, foram encontrados maiores valores durante a ocorrência das chuvas. Compostos como o nitrito e o nitrato apresentaram valores muitos baixos ou não foram determinados.

As oscilações dos valores de alcalinidade e dureza da água podem ser associadas ao regime de chuvas, já que o aumento destes, registrado durante o período de estiagem, foi possivelmente provocado pela maior taxa de evaporação da água, característica desse período, que contribui para o aumento da precipitação de sais (Abílio et al., 2005a). Ao contrário, a ocorrência de precipitação pluviométrica tende a diminuir os valores dessas variáveis, pelo efeito da diluição. Abílio et al. (2005a) também observaram esse tipo de flutuações em estudos por eles realizados em açudes temporários localizados na região semiárida do estado da Paraíba (Brasil).

Tabela 3. Valores médios das variáveis físicas e químicas das águas do rio Batalha no reservatório de captação, nos períodos seco e chuvoso.

\begin{tabular}{l|cc}
\hline Variáveis físicas e químicas & Período Seco & Período Chuvoso \\
\hline Temperatura da água $\left({ }^{\circ} \mathrm{C}\right)$ & 17,0 & 23,7 \\
Oxigênio dissolvido $(\mathrm{mg} / \mathrm{L})$ & 6,0 & 4,0 \\
Condutividade elétrica $(\mu \mathrm{S} / \mathrm{cm})$ & 227,5 & 203,0 \\
$\mathrm{pH}$ & 6,5 & 7,2 \\
Alcalinidade $(\mathrm{mg} / \mathrm{L})$ & 102,2 & 76,4 \\
Dureza total $(\mathrm{mg} / \mathrm{L})$ & 81,0 & 80,2 \\
Amônia $(\mathrm{mg} / \mathrm{L})$ & 0,3 & 0,4 \\
Nitrato $(\mathrm{mg} / \mathrm{L})$ & 0,1 & $\mathrm{ND}$ \\
Nitrito $(\mathrm{mg} / \mathrm{L})$ & $\mathrm{ND}$ & $\mathrm{ND}$ \\
Fosfato $(\mathrm{mg} / \mathrm{L})$ & 1,9 & 1,6 \\
\hline
\end{tabular}

* Não determinado 
De acordo com Johnson et al. (1993), o estado trófico de represas e lagos pode ser caracterizado não somente pelas variáveis ambientais, mas também pela abundância faunística, particularmente, de Oligochaeta e imaturos de Chironomidae, que neste estudo comprovaram que o sistema se encontra em avançado estado de degradação, devido às alterações ambientais consequentes das atividades antrópicas.

Algumas das variáveis químicas da água tais como as concentrações de oxigênio dissolvido e a dureza total parecem associar-se positivamente à diversidade de macroinvertebrados aquáticos. Abílio et al. (2005b), estudando um açude temporário, também encontraram maior diversidade e abundância de insetos aquáticos quando os parâmetros obtidos para essas variáveis se mostravam mais elevados.

\section{CONCLUSÕES}

Neste trabalho, as variações observadas com relação à comunidade estudada - e associadas às alterações ambientais promovidas pela estiagem ou a ocorrência de chuvas mostram a presença de uma fauna bentônica oportunista, constituída, principalmente, por organismos pertencentes às famílias Chironomidae, Tubificidae e Naididae, conhecidas como tolerantes ao enriquecimento orgânico das águas (Strixino e Strixino, 1982), o que sugere que o ambiente estudado se encontra em processo de eutrofização e legitima os resultados obtidos das análises físicas e químicas realizadas no sistema.

\section{AGRADECIMENTOS}

Os autores agradecem à bióloga Tatiani Freitas da Silva pelo grande auxílio no trabalho em campo, ao Departamento de Ciências Biológicas da UNESP de Bauru, bem como à ETADAE/Bauru e seus funcionários.

\section{REFERÊNCIAS}

ABÍLIO, F. J. P.; FONSECA-GESSNER, A. A.; WATANABE, T.; LEITE, R. L. Fauna de Chironomidae e outros insetos aquáticos de açudes do semi-árido paraibano, Brasil. Entomológica y Vectores, v. 12, n. 2, p. 255-264, 2005 a.

ABÍLIO, F. J. P.; FONSECA-GESSNER, A. A.; WATANABE, T.; LEITE, R. L. Chironomus gr. decorus (Diptera: Chironomidae) e outros insetos aquáticos de um açude temporário do semi-árido paraibano, Brasil. Entomológica y Vectores, v. 12, n. 2, p. 233-242. 2005 b.

AMERICAN PUBLIC HEALTH ASSOCIATION - APHA. Standard Methods for examination of water and wastewater. Washington: American Public Health Association, 1995. $1193 \mathrm{p}$.

BRANDIMARTE, A. L. Macrofauna bentônica da zona litoral da Represa de Paraibuna (SP): influência de diferentes ecossistemas terrestres perimetrais. 1991. 161f. Tese (Doutorado em Ciências) - Universidade de São Paulo, São Paulo, 1991.

BRINKHURST, R. O.; MARCHESE, M. R. Guia para la identification de Oligoquetos aquaticos continentales de Sud y Centroamerica. Clímax: Santa Fe, 1989. 207 p. 
CALLISTO, M.; GONÇALVES JR., J. F.; MORENO, P. Invertebrados aquáticos como bioindicadores. In: Navegando o Rio das Velhas das Minas aos Gerais: Proj. Manuelzão. Belo Horizonte: Coopmed, 2005. p. 555-567.

COHEN, A. S. Distribution and faunal associations of benthic invertebrates at Lake Turkuna, Kenya. Hydrobiologia, v. 141, n. 3, p. 179-197, 1986.

DUMNICKA, E. The effect of dam reservoirs on oligochaete communities in River Dunajec (Southern Poland). Acta Hydrobiologica, v. 29, n. 1, 25-34, 1987.

DUMONT, H. J. The species richness of reservoir plankton and effect of reservoirs on plankton dispersal (with particular emphasis on rotifers and cladocerans. In: Theoretical reservoir ecology and its applicatons. São Carlos: Internat. Inst. Ecol./Backhuys Publishers, 1999. p. 477-492.

FERNANDEZ, H. R.; DOMINGUEZ, E. Guía para la determinación de los artrópodos bentónicos sudamericanos. Tucumán: Universidad Nacional de Tucumán, Faculdade de Ciencias Naturales e Instituto Miguel Lillo, 2001. 282p.

FONSECA-GESSNER, A. A.; GUERESCHI, R. M. Macroinvertebrados bentônicos na avaliação da qualidade da água de três córregos na Estação Ecológica de Jataí, Luiz Antonio, SP, Brasil. In: Estudos integrados em ecossistemas: Estação Ecológica de Jataí. São Carlos: Rima, 2000. p.707-731.

FUSARI, L. M. Estudos das comunidades de macroinvertebrados bentônicos das Represas do Monjolinho e do Fazzari no campus da UFSCar, município de São Carlos, SP. 2006. 88f. Dissertação (Mestrado em Ecologia e Recursos Naturais) Universidade Federal de São Carlos, São Carlos, 2006.

GOLTERMAN, H. L.; CLYMO, R.S.; OHNSTAD, M. A. M. Methods for physical and chemical analysis of freshwater. Oxford: Blackwell Scientific Publications, 1978. 214 p.

HAUER, F. R.; RESH, V. H. Benthic macroinvertebrates. San Diego: Academic Press, 1996. $684 \mathrm{p}$.

JOHNSON, R. K.; WIEDERHOLM, T.; ROSENBERG, D. M. Freshwater biomonitoring using individual organisms, populations, and species assemblages of benthic macroinvertebrates. In: Freshwater biomonitoring and benthics macroinvertebrates. New York: Chapman \& Hall, 1993. p. 40-158.

KLEINE, P.; TRIVINHO-STRIXINO, S. Chironomidae and other aquatic macroinvertebrates of a first order stream: community response after habitat fragmentation. Acta Limnologica Brasiliense, v. 17, n. 1, p. 81-90, 2005.

LAKE, P. S. Disturbing hard and soft bottom communities: a comparison of marine and freshwater environments. Australian Journal of Ecology, v. 15, p. 477-488, 1990.

LINDEGAARD, C. Classification of water-bodies and pollution. In: The Chironomidae: biology and ecology of non-biting midges. London: Chapman \& Hall, 1995. p. 385-404.

MACKERETH, F. J. H.; HERON, J.; TALLING, J. F. Water analysis: some revised methods for limnologists. Titus: Kendal, 1978. $121 \mathrm{p}$.

MERRIT, R.; CUMMINS, K. An introduction to the aquatic insects of North America. Dubuque: Kendall Hunt, 1996. 722 p. 
SILVA, F. L.; TALAMONI, J. L. B.; BOCHINI, G. L.; RUIZ, S. S.; MOREIRA, D. C. Macroinvertebrados aquáticos do reservatório do rio Batalha para a captação das águas e abastecimento do município de Bauru, SP, Brasil. Ambi-Agua, Taubaté, v. 4, n. 2, p. 66-74, 2009. (doi:10.4136/ambi-agua.87)

PINTO-COELHO, R. M. Fundamentos em Ecologia. Porto Alegre : Artmed, 2002. 252 p.

QUEIROZ, J. F.; TRIVINHO-STRIXINO, S.; NASCIMENTO, V. M. C. Organismos bentônicos bioindicadores da qualidade de água da bacia do médio São Francisco. Embrapa Meio Ambiente, v. 3, p. 1-4, 2000.

RODIER, J. L'analyse de l'eau: eaux naturelles, euax résiduales, eaux de mer. Paris: Dunod, 1975. $692 \mathrm{p}$.

RODRIGUES, M. H. S. Estudo da fauna de Chironomidae (Diptera) do sedimento na represa do Beija-Flor, na Estação de Jataí, Luís Antônio, SP. 1997. 85f. Dissertação (Mestrado em Ecologia e Recursos Naturais) - Universidade Federal de São Carlos, São Carlos, 1997.

ROQUE, F. O.; TRIVINHO-STRIXINO, S. Avaliação preliminar da qualidade da água dos córregos do município de Luis Antônio (SP) utilizando macroinvertebrados bentônicos como bioindicadores: subsídios para o monitoramento ambiental. Ciências Biológicas e do Ambiente, v. 2, n. 1, p. 21-34, 2000.

SHIMIZU, G. Y. Represa de Americana: aspectos dos bentos litoral. 1978. 148f. Tese (Doutorado em Ciências) - Universidade de São Paulo, São Paulo, 1978.

STRIXINO, G. ; STRIXINO, S. T. Macrobentos da Represa do Monjolinho (São Carlos - SP). Revista Brasileira de Biologia, v. 42, n. 1, p. 165-170, 1982.

STRIXINO, G.; TRIVINHO-STRIXINO, S. Povoamentos de Chironomidae (Diptera) em lagos artificiais. Oecologia Brasiliensis, v. 5, p. 141-154, 1998.

TALAMONI, J. L. B. Estudo das características físicas, químicas e bióticas da bacia hidrográfica do rio Batalha (sub-bacia do médio Tietê). In: CONGRESSO DE EXTENSÃO UNIVERSITÁRIA DA UNESP, 1., 1999, São Pedro, Anais... São Pedro: UNESP, Pró-Reitoria de Extensão, 1999. p. 1-3.

TUNDISI, J. G. Ambiente, represas e barragens. Ciência Hoje, v. 1, p. 41-461, 1992. 